\title{
The CORALS survey
}

\section{Clues to galaxy clustering around QSOs from $z_{\mathrm{abs}} \sim z_{\mathrm{em}}$ damped Lyman alpha systems ${ }^{\star}$}

\author{
S. L. Ellison ${ }^{1}$, L. Yan ${ }^{2}$, I. M. Hook ${ }^{3}$, M. Pettini ${ }^{4}$, J. V. Wall ${ }^{5}$, and P. Shaver ${ }^{6}$ \\ 1 European Southern Observatory, Casilla 19001, Santiago 19, Chile \\ 2 SIRTF Science Center, Caltech, California, USA \\ e-mail: lyan@ipac.caltech.edu \\ 3 Astrophysics: Department of Physics, Nuclear and Astrophysics Laboratory, Keble Road, \\ Oxford OX1 3RH, UK \\ e-mail: ihook@gemini.edu \\ 4 Institute of Astronomy, Madingley Rd., Cambridge CB3 0HA, UK \\ e-mail: pettini@ast.cam.ac.uk \\ 5 Astrophysics: Department of Physics, Nuclear and Astrophysics Laboratory, Keble Road, \\ Oxford OX1 3RH, UK \\ e-mail: jvw@astro.ox.ac.uk \\ 6 European Southern Observatory, Karl-Schwarzschild-Str. 2, 85748 Garching bei Munchen, Germany \\ e-mail: pshaver@eso.org
}

Received 14 August 2001 / Accepted 4 December 2001

\begin{abstract}
We present a list of $z_{\mathrm{abs}} \sim z_{\mathrm{em}}\left(\Delta v<3000 \mathrm{~km} \mathrm{~s}^{-1}\right)$ DLAs discovered during the CORALS survey for absorbers in a radio-selected QSO sample. On the assumption that $z_{\mathrm{abs}} \sim z_{\mathrm{em}}$ DLAs are neither ejecta from the QSO, nor part of the host galaxy itself, we use the number density statistics of these DLAs to investigate galaxy clustering near the QSO redshift. We find that $n(z)$ of $z_{\mathrm{abs}} \sim z_{\mathrm{em}}$ DLAs in our radio-selected QSO sample is $\sim 4$ times larger than the number density of intervening DLAs, implying an excess of galaxies near the QSO. This result is further supported with the inclusion of the radio-loud QSOs in the FIRST survey, although the total number of DLAs is still small (4) and the result is currently only significant at approximately the $2 \sigma$ level. Since all of the $z_{\mathrm{abs}} \sim z_{\mathrm{em}}$ DLAs we identify in CORALS are found towards optically bright $(B<20)$ QSOs, there is no strong evidence (based on these limited statistics) that this population suffers from a severe dust bias. We compare our results with those from an optically-selected, radio-quiet QSO sample in order to determine whether there is evidence for an excess of galaxies around radio-loud versus radio-quiet QSOs. We find that the $n(z)$ of $z_{\mathrm{abs}} \sim z_{\mathrm{em}}$ DLAs towards radio-quiet QSOs is in agreement with the number density of intervening absorbers. This result, although currently limited by the small number statistics of our survey, supports the conclusion that radio-loud quasars are found preferentially in rich galaxy environments at high redshift. Finally, we report that one of the new $z_{\text {abs }} \sim z_{\mathrm{em}}$ DLAs discovered by CORALS has some residual flux in the base of the Lyman $\alpha$ trough which may be due to Lyman $\alpha$ emission, either from star formation in the DLA galaxy or from gas photoionised by the QSO.
\end{abstract}

Key words. quasars: general - quasars: absorption lines - galaxies: evolution - galaxies: clusters: general

\section{Introduction}

The formation of quasars is believed to represent an integral stage in the process of galaxy formation and evolution. The high incidence of galaxy-QSO companions and apparent mergers supports the view that quasar activity

Send offprint requests to: S. Ellison,

e-mail: sellison@eso.org

* The work presented here is based, in part, on data obtained with the ESO facilities on La Silla (EFOSC/3.6-m) and Paranal (FORS1/UT1), programs 66.A-0037 and 267.A-5686. is fuelled by continued accretion of material onto the central black hole from the intercluster medium or nearby galaxies (see the review by Barnes \& Hernquist 1992). Many of the outstanding questions in theories of the role of AGN in galaxy evolution involve the link between radio power and the properties of the QSO environment. The long-standing view is that both the morphology of the host galaxy (e.g. Hamilton et al. 2001), and the richness of the surrounding environment (Yee \& Green 1987; Hall \& Green 1998; Hall et al. 1998; Hutchings et al. 1999; 
Teplitz et al. 1999; Cimatti et al. 2000) are different between radio-quiet QSOs (RQQs) and radio-loud QSOs (RLQs). Specifically, Yee \& Green (1987) pointed out that the former are rarely found in clusters, while about $35 \%$ of RLQs show an excess of faint galaxies in their vicinity, consistent with the presence of a cluster of galaxies at the QSO redshift (see also Sanchez \& Gonzalez-Serrano 1999). Radio galaxies, which are intimately connected with RLQs in unification models, also appear to reside in cluster environments at high redshift (Pentericci et al. 2000). However, the dichotomy between radio-loud and radio-quiet properties is now being questioned by studies which have found that almost all QSO hosts are elliptical galaxies, regardless of radio-loudness (McLure et al. 1999; Dunlop et al. 2002), and that the environments of RLQs are statistically indistinguishable from those of RQQs, at least at $z \lesssim 1$ (Hutchings et al. 1995; McLure \& Dunlop 2001; Wold et al. 2001; Finn et al. 2001). At higher redshifts, there is evidence that galaxy density maybe higher around RLQs, although a milder overdensity is seen towards RQQs (see review by Hutchings 2001). This ongoing discussion is made even more relevant by (a) our lack of understanding of the mechanism responsible for the radio power of RLQs and (b) the recent paradigm shift away from the long held belief that the distribution of radio luminosities is bimodal (Kellermann et al 1989; White et al. 2000; Brinkmann et al. 2000; Lacy et al. 2001). It is of primary importance to establish the extent to which radio power depends on the QSO environment, since this relationship clearly holds important clues to the physics of AGN and the role of quasars in galaxy formation.

In this paper we explore the possibility of using quasar absorption lines as an unbiased probe of the high redshift QSO environment. This is a potentially powerful technique since studies of galaxy clustering around QSOs can be extended to much higher redshifts, although the obvious disadvantage is the uni-dimensional information content. Therefore, a large number of QSOs needs to be studied in order for the statistical trends of $z_{\text {abs }} \sim$ $z_{\mathrm{em}}$ absorbers to provide meaningful results. In this respect, the results presented here for the 66 QSOs of the Complete Optical and Radio Absorption Line System survey (CORALS, Ellison et al. 2001b) should be regarded as preliminary, although they underline the potential of this approach, so far relatively unexploited.

There is already some evidence that the posited correlation between galaxy concentration and radio-loudness may extend to QSO absorption line systems, based on an excess of associated C IV systems (Foltz et al. 1986; Anderson et al. 1987; Foltz et al. 1988) in RLQ spectra out to $3000 \mathrm{~km} \mathrm{~s}^{-1}$ from the QSO redshift. However, the origin of these absorbers is unclear. The excess seems to be restricted to steep-spectrum RLQs, and may thus be related to some orientation effect (Barthel et al. 1997; Richards et al. 1999; Richards 2001). On the other hand, Richards et al. (2001) find no correlation of this excess with other orientation measures such as core-to-lobe ratio. Nevertheless, the suspicion remains that these unusually strong and highly ionised systems are probably intrinsic to the QSO and may not be providing us with specific clues about the surrounding galaxy environment (Baker et al 2001). A more fruitful line of investigation is to focus on $z_{\text {abs }} \sim z_{\text {em }}$ absorbers which are analogous to galaxy-scale absorbers at intervening redshifts, namely the Damped Lyman Alpha systems (DLAs).

In the last 15 years, several surveys have mined the sky for DLAs, with the objective of understanding more about the high redshift galaxy population that these absorption systems are thought to represent (Wolfe et al. 1986; Lanzetta et al. 1991, 1995; Wolfe et al. 1995; StorrieLombardi \& Wolfe 2000; Péroux et al. 2001). Almost without exception, these surveys have adopted the early strategy and definitions of Wolfe et al. (1986) who included in their sample only DLAs with $N(\mathrm{H} \mathrm{I}) \geq 2 \times 10^{20}$ atoms $\mathrm{cm}^{-2}$ that lie at absorption redshifts corresponding to $\Delta v>3000 \mathrm{~km} \mathrm{~s}^{-1}$ from the QSO emission redshift. The reason for imposing this velocity cut is primarily due to the unknown nature of these proximate DLAs (PDLAs), which may conceivably be caused by QSO ejecta or by absorption in the host galaxy itself. In addition, since one of the main objectives of DLA surveys has been to compile a census of $\mathrm{H}$ I in the universe, proximate systems have been excluded due to the possible effect of the QSO's ionizing radiation on the absorber. For example, the intense local radiation field of the QSO is already well known to affect the distribution of the lower column density Lyman $\alpha$ forest clouds (e.g. Murdoch et al. 1986). A similar phenomenon has also been noted to affect the extended Lyman $\alpha$ haloes of low redshift galaxies (Pascarelle et al. 2001). Therefore, in the quest to gain an insight into the "normal and representative" galaxy population at high redshift, PDLAs at $z_{\text {abs }} \sim z_{\text {em }}$ have been almost universally discarded from the statistics of previous surveys.

Møller et al. (1998), motivated by observations of Lyman $\alpha$ emission in associated DLAs, have explored the possible nature of these systems. They concluded that PDLAs are unlikely to be due to QSO ejecta, based on their lower metallicities, lack of strong high ionization lines and simpler line profiles compared to intrinsic BAL features (see Barlow et al. 1997 for a summary of intrinsic absorber signatures). Although recent models for the structure of AGN (e.g. Elvis 2000) include relatively high column density, wind-ejected clouds, the consequent UV absorbers are quite different from PDLAs both in terms of $N(\mathrm{H} \mathrm{I})$ and ejection velocity (e.g. Monier et al. 2001). For example, narrow intrinsic C IV systems, identified by means of time variability or partial coverage have relatively low H I column densities (e.g. Petitjean et al. 1994; Hamann et al. 1997). It is also unlikely that PDLAs are generally due to the QSO host galaxy, again because of the significant velocity differences involved. It therefore seems highly plausible that PDLAs are either members of the same family as, or at least strongly resemble, the population of intervening absorbers. If this interpretation holds true, then investigating the hitherto unexploited sample 


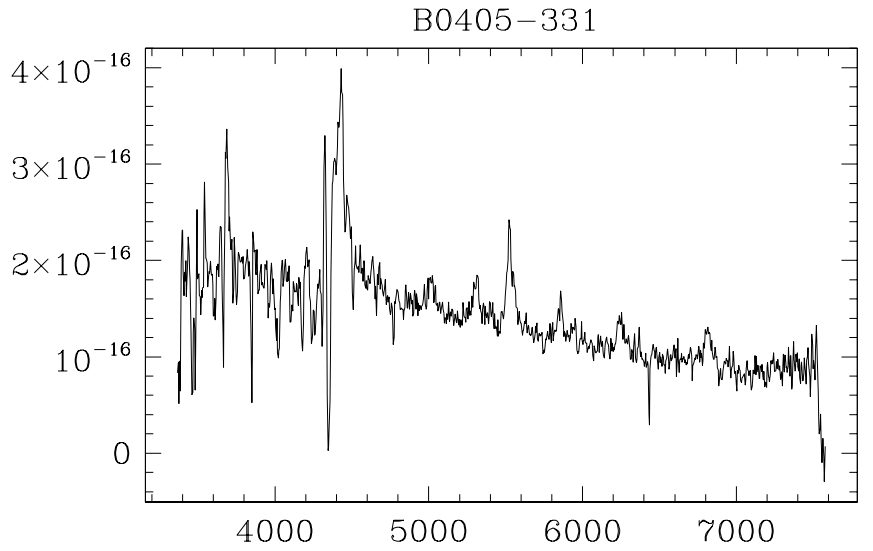

B1354-107

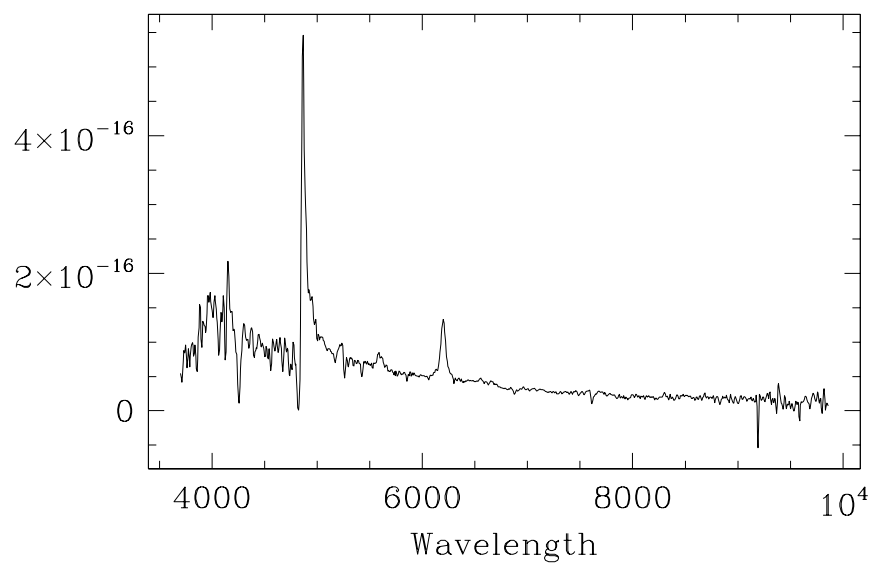

Fig. 1. Discovery spectra (obtained with EFOSC at the 3.6-m on La Silla) of two of the CORALS QSOs with proximate DLAs showing that these sources are not BAL QSOs. A third QSO with a PDLA, B0528-250, was already known and was not reobserved.

of PDLAs may yield further insights into the connection between AGN activity and galaxy formation.

Based on a radio-selected sample of QSOs, CORALS (Ellison et al. 2001b) is one of the most recent DLA surveys aimed at determining the extent of any dust bias affecting the statistics of DLAs drawn from opticallyselected, magnitude limited QSO samples. In this paper, we present the PDLAs found by the CORALS survey (Sect. 2) and compare their rate of incidence with that of intervening absorbers at $\Delta v>3000 \mathrm{~km} \mathrm{~s}^{-1}$ in the same sample of QSOs (Sect. 3). We also compare the frequency of PDLAs between RLQs and RQQs. Our main result is that we find a possible excess of PDLAs towards RLQs (currently only confirmed at slightly below the $2 \sigma$ level), and that since all of the systems that we identify lie in front of optically bright QSOs it appears that PDLAs contain only a modest amount of dust. These findings, which are still tentative because of the small size of present samples, are briefly discussed in Sect. 4 .

\section{Identified PDLAs in CORALS}

Full details of the CORALS sample definition, observations and all spectra can be found in Ellison et al. (2001b). In brief, a sample of $66 z_{\mathrm{em}} \geq 2.2$ radio-loud
QSOs was selected from the flat-spectrum $(\alpha>-0.4)$ sources in PKSCat90, the catalogue compendium from the Parkes surveys. A full list of flux densities, source positions and spectral indices is given Jackson et al. (2002). For this radio-selected sample, an optical spectrum was obtained for each QSO in order to search for DLAs with $1.8<z_{\mathrm{abs}}<z_{\mathrm{em}}$. Out of a total of 22 confirmed DLAs, three were at redshifts corresponding to velocities $\Delta v \leq$ $3000 \mathrm{~km} \mathrm{~s}^{-1}$ (the original limit adopted by Wolfe et al. 1986). We note that the Lyman $\alpha$ line used here to determine $z_{\mathrm{em}}$ may be systematically blue-shifted with respect to other emission lines, implying that the actual velocity offset of the PDLAs maybe be up to $\sim 4500 \mathrm{~km} \mathrm{~s}^{-1}$ from $z_{\mathrm{em}}$. However, this does not affect our analysis because the $3000 \mathrm{~km} \mathrm{~s}^{-1}$ limit traditionally adopted to distinguish intervening systems is measured relative to Ly $\alpha$, not $\mathrm{H} \alpha$ or any highly ionized lines. The three PDLAs are in the sightlines towards B1354-107b, B0405-331 and B0528-250. The third of these systems has been known for a long time - Morton et al. (1980) measured $z_{\text {abs }}=2.811$ and $N(\mathrm{H} \mathrm{I})=1.6 \times 10^{21} \mathrm{~cm}^{-2}-$ and was therefore not reobserved as part of the CORALS survey. In Fig. 1 we present the original low resolution spectra of the other 2 QSOs found to exhibit PDLAs, obtained to confirm the QSO identification and redshift. The large wavelength coverage out to beyond C IV allows us to confirm that these are not BAL QSOs and that the PDLAs are therefore unlikely to be high velocity ejected material. Higher resolution ( $F W H M \sim 3 \AA$ ) spectra have been obtained of these targets with the AAT, see Ellison et al. (2001b) for spectra and details of observation and data reduction. Figure 2 shows close-ups of the damped Lyman $\alpha$ lines together with adopted fits to the line profiles.

We will now briefly describe the properties of these two new systems.

B1354-107b Two DLAs are detected in the sightline towards this QSO, the higher redshift absorber "b" having a redshift of $z_{\mathrm{abs}}=2.966$. The velocity difference between the absorber and the emission redshifts $\left(z_{\mathrm{em}}=3.006\right)$ is therefore $\sim 3000 \mathrm{~km} \mathrm{~s}^{-1}$, just on the limit of our definition of a PDLA given the uncertainty of $z_{\mathrm{em}}$ and $z_{\mathrm{abs}}$. The Lyman limit at $\lambda_{0}=912 \AA$ associated with this system is clearly visible at $\lambda_{\text {obs }} \sim 3650 \AA$ in our AAT spectrum (see Ellison et al. 2001b). The H I fit to this absorber is complicated by the onset of Lyman $\alpha$ emission in the absorber's red wing. No information about the column density can therefore be gleaned from this part of the line profile. There is some additional, blended absorption in the blue, so the fit is mainly constrained by the base of the trough, see Fig. 2.

B0405-331 This DLA has an absorption redshift $z_{\mathrm{abs}} \sim$ 2.57 which is the same as the QSO redshift of $z_{\mathrm{em}}=2.570$. The absorption trough of this system is superimposed on the Lyman $\alpha$ emission from the quasar itself, rendering an accurate continuum fit very difficult. Figure 2 (middle 
$\mathrm{B} 1354-107 \mathrm{~N}(\mathrm{HI})=2.5 \mathrm{e} 20$
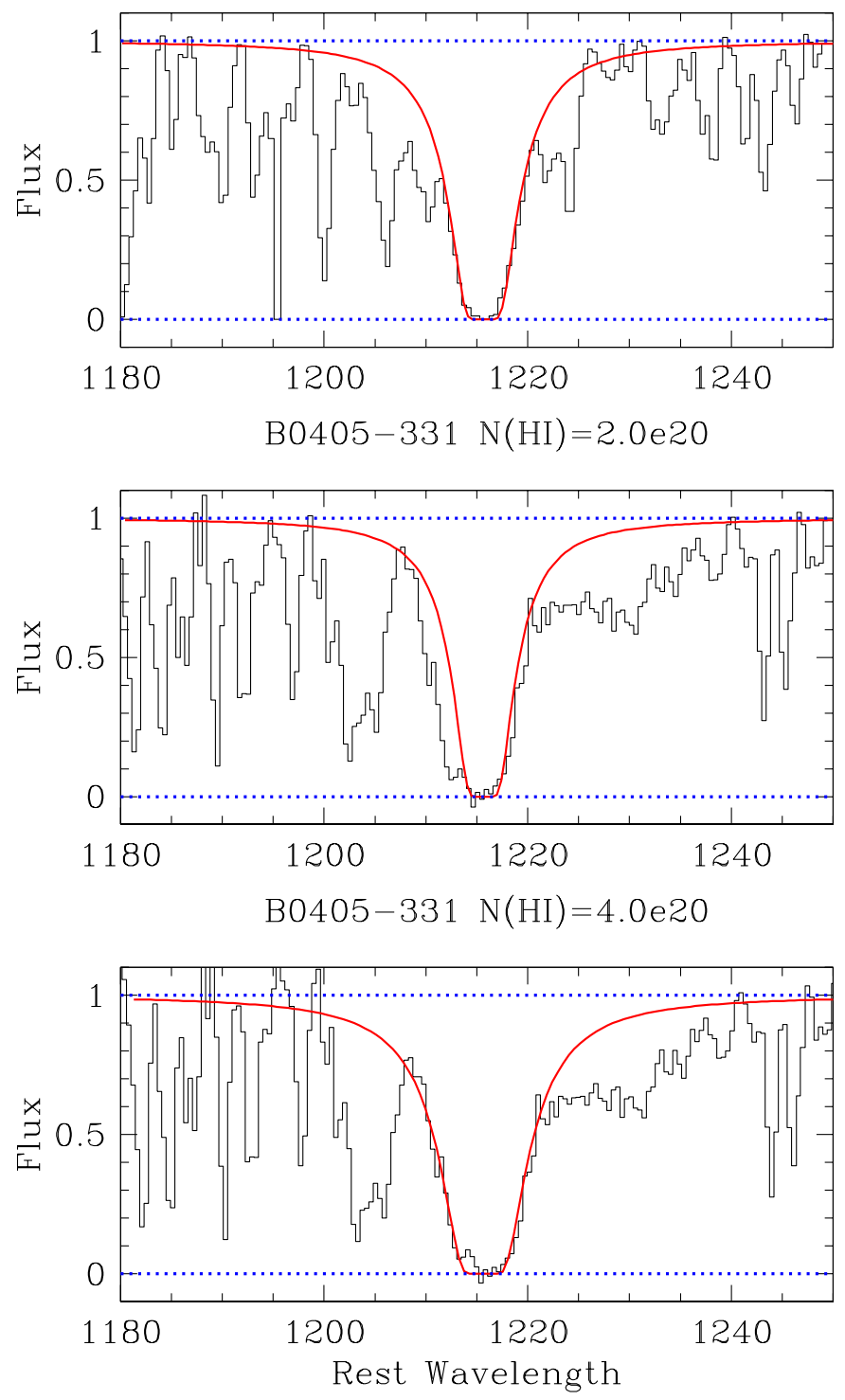

Fig. 2. H I fits to the 2 new PDLAs found in the CORALS sample. Top panel: fit to the $z_{\text {abs }}=2.966$ DLA towards B1354-107. Middle panel: possible fit to the DLA towards B0405-331 with $z_{\text {abs }}=2.572$ and $N(\mathrm{H} \mathrm{I})=2 \times 10^{20} \mathrm{~cm}^{-2}$. Bottom panel: alternative fit to the DLA towards B0405-331 with $z_{\mathrm{abs}}=2.570$ and $N(\mathrm{H} \mathrm{I})=4 \times 10^{20} \mathrm{~cm}^{-2}$. In this case, some residual flux can be seen at the blue edge of the Lyman $\alpha$ trough.

panel) shows that a good fit to the damped Lyman $\alpha$ line is obtained with a $N(\mathrm{H} \mathrm{I})=2 \times 10^{20} \mathrm{~cm}^{-2}$ at $z_{\text {abs }}=2.572^{1}$. However, we find that an absorber at $z_{\text {abs }}=2.570$ and with $N(\mathrm{H} \mathrm{I})=4 \times 10^{20} \mathrm{~cm}^{-2}$ would also produce a good fit to the base and the blue slope of the absorption profile, although there is a small but significant residual flux

\footnotetext{
${ }^{1}$ Although this is nominally higher than the emission redshift, the difference is only $168 \mathrm{~km} \mathrm{~s}^{-1}$, well within the uncertainty in the QSO systemic redshift and within the velocity dispersion of a galaxy cluster of which the QSO host galaxy may be a member.
}

feature visible in the wing of the trough (bottom panel of Fig. 2). Several associated metal lines are present in our spectrum, particularly C II $\lambda 1334$, Si II $\lambda 1260$ and Si II $\lambda 1190$ with redshifts of $z_{\text {abs }}=2.569,2.569$ and 2.570 respectively. Lyman $\beta$ is also covered by these observations and is found at a redshift of 2.570. These lines therefore more closely match the lower redshift, higher column density solution shown in the bottom panel of Fig. 2. This would then leave a small residual flux in the base of the DLA absorption which we interpret as Lyman $\alpha$ emission associated with the absorbing galaxy. Its velocity offest from the centre of the absorption trough is $\Delta v=-300 \mathrm{~km} \mathrm{~s}^{-1}$, very similar to that between the DLA and Lyman $\alpha$ emitter in Q0151+048A (Møller et al. 1998; Fynbo et al. 1999).

There is no obvious CCD defect or cosmic ray event at the position of the residual flux feature on the $2 \mathrm{D}$ spectrum. This is clearly a system which should be followed up with narrow band imaging as it would add to the handful of known DLAs which have associated Lyman $\alpha$ emission. It is interesting to note that there is some evidence that PDLAs are more likely to exhibit strong Lyman $\alpha$ emission than $z_{\text {abs }} \ll z_{\text {em }}$ "intervening" DLAs (Møller et al. 1998). Although the presence of Lyman $\alpha$ emission may in some cases be caused by photo-ionization (e.g. Fynbo et al. 1999; Warren et al. 2001), there is also evidence that star formation may be involved in other cases (e.g. Møller \& Warren 1996; Ellison et al. 2001a).

\section{Results}

\subsection{The number density of PDLAs in the radio-loud CORALS sample}

The statistics of our survey are collected in Table 1. The number of damped absorbers found per unit redshift can be expressed as the DLA number density, $n(z)$, quoted for a specific mean absorption redshift. This is simply the total number of DLAs identified in a given sample, divided by the total intervening redshift interval covered. The redshift interval, $\Delta z$, is given by

$\Delta z=\sum_{i=1}^{n}\left(z_{i}^{\max }-z_{i}^{\min }\right)$

where the summation is over the $n$ QSOs in a given sample. In the case of PDLAs, we only include the redshift interval from the emission redshift extending out to $3000 \mathrm{~km} \mathrm{~s}^{-1}$,

$\Delta z_{3000}=\sum_{i=1}^{n}\left(z_{i}^{\mathrm{em}}-z_{i}^{3000}\right)$

where $z_{i}^{3000}$ is the redshift corresponding to a relative velocity of $3000 \mathrm{~km} \mathrm{~s}^{-1}$.

For the CORALS survey, we find $n(z)=1.2_{-0.7}^{+1.2}$ at a mean $\langle z\rangle=2.78$ (using $1 \sigma$ confidence limits appropriate to the Poissonian small number statistics formulation by Gehrels 1986). This value is $4_{-2.4}^{+4}$ times larger than 
Table 1. Number density statistics for PDLA samples in CORALS, the FBQS survey (White et al. 2000) and the survey of Péroux et al. (2001). The last column gives the expected number density of PDLAs according to the statistics for intervening DLAs determined by Storrie-Lombardi \& Wolfe (2000).

\begin{tabular}{lccccccc}
\hline \hline Sample & No. QSOs & QSO Type & No. DLAs & $\Delta z_{3000}$ & $n(z)$ PDLA & $\left\langle z_{\text {abs }}\right\rangle$ & $n(z)$ DLA \\
\hline CORALS & 66 & RLQ & 3 & 2.48 & $1.21_{-0.65}^{+1.17}$ & 2.78 & 0.24 \\
CORALS+FBQS & 96 & RLQ & 4 & 3.58 & $1.12_{-0.54}^{+0.88}$ & 2.76 & 0.24 \\
Péroux et al. & 49 & RQQ & 1 & 2.56 & $0.39_{-0.31}^{+0.35}$ & 4.26 & 0.35 \\
\hline
\end{tabular}

value $n(z)=0.31_{-0.08}^{+0.09}$ at $\langle z\rangle=2.37$ determined for the intervening DLAs in the CORALS sample by Ellison et al. (2001b). We find a similar apparent excess compared to the density of intervening DLAs determined from optical samples. Adopting $n(z)=0.055(1+z)^{1.11}$, as deduced by Storrie-Lombardi \& Wolfe (2000), we would have expected only $n(z)=0.24$ at $\langle z\rangle=2.78$. However, we are dealing with very small number statistics here. Since the redshift path $\Delta z$ which can be probed for PDLAs in a given QSO sight-line is very small, a much larger sample is required to establish conclusively if PDLAs are indeed more common towards RLQs.

In an effort to improve the statistics, we also consider the recent radio-selected FIRST Bright Quasar Survey (FBQS; White et al. 2000). Specifically, we selected the 41 QSOs with $z_{\mathrm{em}} \geq 2.2$ in this independent sample (excluding BALs). Due to the different selection criteria and survey sensitivities of the FBQS compared to CORALS, we further exclude the 11 FBQS QSOs that do not conform to the accepted definition of radio-loud, i.e. with a radioloudness parameter $\log R^{*} \geq 1.0$ (Stocke et al. 1992). Combining the remaining 30 FBQS targets (with 1 PDLA) with CORALS, we cover a total interval $\Delta z_{3000}=3.58$ and deduce $1.1_{-0.5}^{+0.9}$. Thus, although the errors are still large, these statistics indicate that there may be an excess of absorbers close to the systemic redshift of radio-loud QSOs. If the postulate that PDLAs are not directly associated with the QSO is true (Møller et al. 1998), then this excess may be analogous to the excess of galaxies observed in the fields of RLQs (Yamada et al. 1997; Hall \& Green 1998; Hall et al. 1998; Clements 2000). Indeed, the excess of DLAs close to the QSO compared with intervening systems is consistent with the typical over-densities of galaxies found around RLQs by Sanchez \& GonzalezSerrano (1999) and of Lyman $\alpha$ emitters around radio galaxies (Kurk et al. 2001).

\subsection{Comparison of the number density of $D L A$ s between $R L Q s$ and $R Q Q s$}

If PDLAs are associated with galaxies clustering around QSOs, then comparing the properties of these absorbers in radio- versus optically-selected QSO sample will provide further evidence as to whether RLQs preferentially flag rich cluster environments at high redshift. However, this is not a simple undertaking for the following reason. Most of the early DLA surveys initially obtained low resolution (typically $10 \AA$ ) spectra of all the QSOs in a given sample.
From this, a list of candidate DLAs with rest frame equivalent width $W_{0} \geq 5 \AA$ was compiled and subsequently followed up at higher resolution to confirm the DLA nature and measure the column density $N(\mathrm{H} \mathrm{I})$. However, in most cases PDLAs were not included in the follow-up spectroscopy. Ellison (2000) investigated how the probability that an absorption system is damped varies as a function of $W_{0}$ and found that $\sim 90 \%$ of DLA candidates with $W_{0}>10 \AA$ were eventually confirmed to have $N(\mathrm{H} \mathrm{I}) \geq 2 \times 10^{20} \mathrm{~cm}^{-2}$. Therefore, it is in principle possible to use these statistical likelihoods to infer the number of PDLAs in the previous major surveys. However, since PDLAs have been universally excluded from these surveys, even the equivalent widths have generally not been tabulated in the literature. Given this unsatisfactory state of affairs, here we restrict ourselves to comparing our estimate of $n(z)$ of PDLAs in CORALS with analogous statistics from the recent survey by Péroux et al. (2001).

From the Péroux et al. (2001) sample of 66 QSOs, we exclude all targets which exhibit BAL features (10) or are confirmed to be radio-loud (7) based on observations from NVSS and SUMSS measurements. From the remaining 49 QSOs, only 1 PDLA is found ( $z_{\text {abs }}=4.26$ towards PSS J0034+1639). The total redshift interval covered is $\Delta z_{3000}=2.56$ (Péroux, private communication), yielding a mean $n(z)=0.39$ at $\langle z\rangle=4.26$, in good agreement with Storrie-Lombardi \& Wolfe's (2000) derived $n(z)=0.35$ for intervening DLAs at this redshift. Note that despite the difference in mean absorption redshifts between the RLQ and RQQ samples, cluster evolution is unlikely to explain the disparate $n(z)$ due to the small time $(\sim 0.5 \mathrm{Gyr})$ between these two epochs ${ }^{2}$. It therefore appears that the tentatively identified overdensity of DLAs near to the QSO is confined to $R L Q s$.

\subsection{Dust extinction in PDLAs}

Studies of optical (Outram et al. 2001) and IR (Hewett, private communication) colours of large, optically-selected QSO samples find that the effect of dust reddening in intervening absorbers is small. Conversely,

\footnotetext{
${ }^{2}$ We note that out of the 49 QSOs considered in these statistics, 4 have no radio data available. Since only approximately $10 \%$ of QSOs are radio-loud, we make the assumption that none of these 4 targets is radio-loud. Even in the extreme and unlikely case that ALL of these 4 QSOs turned out to be radio-loud, $\Delta z$ would be reduced by less than $10 \%$ to 2.35 , i.e. $n(z)=0.42$, a negligible change.
} 
Carilli et al. (1998) found that a high fraction of "red" QSOs have associated $z_{\text {abs }} \sim z_{\mathrm{em}} 21 \mathrm{~cm}$ absorption, indicating that dust reddening may play a significant effect in proximate absorbers. Consequently, we may expect that optically-selected, magnitude-limited QSO samples may be biased against the detection of PDLAs. It is therefore somewhat surprising that all of the PDLAs of the CORALS sample are found towards relatively bright QSOs $(B<20)$. Taken at face value, this implies that any dust extinction which may be present does not bias the statistics significantly, although it must be remembered that we are dealing with very small number statistics. Since Ellison et al. (2001b) find that up to twice as many intervening DLAs are present towards faint QSOs, it will be interesting to see whether observing more QSOs reveals a similar effect for proximate DLAs. This would establish whether PDLAs are relatively dust-poor or dust-rich.

\section{Conclusions and discussion}

The main aim of this paper has been to assess whether PDLAs can provide an insight into the the clustering of galaxies around QSOs at high redshifts.

The main conclusions of this work may be summarised as follows.

1. Despite the small number statistics of this work (4 DLAs in the combined CORALS + FBQS sample), we find evidence that our radio-selected sample of QSO exhibits an excess (by a factor of 4) of PDLAs at $z_{\text {abs }} \sim z_{\text {em }}$ compared to intervening redshifts. This implies an excess of material (presumably galaxies) near the QSO at levels consistent with faint galaxy excesses around RLQs (e.g. Sanchez \& Gonzalez-Serrano 1999). At present this result is only significant at the $\sim 2 \sigma$ level, due to the limited statistics. However, it is certainly suggestive and emphasises the potential use of proximate DLAs as probes of the environment of QSOs.

2. Similarly, the number density of PDLAs towards RLQs is $>4$ times higher than that of PDLAs towards RQQs. In fact, we find that $n(z)$ of PDLAs towards RQQs is indistinguishable from that of intervening DLAs. This result is consistent with the premise that RLQs preferentially mark cluster environments.

3. Since all of the PDLAs in the CORALS sample are found towards optically bright QSOs $(B<20)$ there appears to be no strong extinction effect from dust. Although previous studies find that dust in $z_{\mathrm{abs}} \sim z_{\mathrm{em}}$ systems may redden the background QSO (Carilli et al. 1998), it appears that the amount of dust that is present is modest and not sufficient to exclude a significant number of quasars from optical surveys.

4. We identify a new PDLA in the line of sight towards B0405-331 which may exhibit Lyman $\alpha$ emission, based on residual flux seen at the base of the saturated absorption trough.
Lacy et al. (2001) have recently proposed a unified scheme for radio-loud and radio-quiet QSOs by showing that the radio luminosity of QSOs scales with the black hole mass and the accretion rate onto the black hole. Although the data support the broad increase in radio luminosity with black hole mass, it is unclear whether accretion rate is the physical driver of this relation (Dunlop et al. 2002). We have presented in this paper evidence suggesting that radio-loud QSOs are found preferentially in richer environments than radio-quiet QSOs. At first sight, such a connection between the pc scale of the central engine and the Mpc scale of galaxy clusters may seem surprising. However, it may simply be another manifestation of the relationship uncovered by Magorrian et al. (1998) between black hole mass and bulge mass, if the most massive galaxies are also more likely to be found in rich clusters.

With the current limited statistics for PDLAs it is difficult to extend the analysis of the DLA environment around QSOs. As the number of identified $z_{\text {abs }} \sim z_{\text {em }}$ systems increases (several candidates should be present in existing surveys) it will be possible to compare the 1-D QSO-DLA correlation function with the galaxy-QSO correlation function in clusters (Martínez et al. 1999). Such a comparison will provide further clues to the nature of PDLAs, and in turn contribute greatly to the investigation of QSO cluster environments. Of course, we must bear in mind that the $3000 \mathrm{~km} \mathrm{~s}^{-1}$ cut-off adopted as the definition for associated DLAs is somewhat arbitrary. This point may be especially important since one of the CORALS PDLAs is right on the $3000 \mathrm{~km} \mathrm{~s}^{-1}$ cut-off. With improved statistics, it will be very interesting to investigate $n(z)$ as a function of relative velocity from the QSO.

Acknowledgements. We are grateful to Céline Péroux for communicating the statistics of PDLAs from the survey of Péroux et al. (2001) and to Dick Hunstead for checking the SUMSS fields of some of these QSOs for their radio properties. Thanks also are due to Marcin Sawicki and Gabriela Mallen-Ornelas for continued stimulating discussions.

\section{References}

Anderson, S. F., Weymann, R. J., Foltz, C. B., \& Chaffee, F. H. 1987, ApJ, 94, 278

Baker J. C., Hunstead, R. W., Athreya, R. M., et al. 2001, ApJ, submitted

Barnes, J. E., \& Hernquist, L. 1992, ARA\&A, 30, 705

Barthel, P. D., Tytler, D. R., \& Vestergaard, M. 1997, Mass Ejection from Active Galactic Nuclei, ASP Conf. Ser., 128, 48

Brinkmann, W., Laurent-Muehleisen, S. A., Voges, W., et al. 2000, A\&A, 356, 445

Carilli, C. L., Menten, K. M., Reid, M. J., Rupen, M. P., \& Yun, M. 1998, ApJ, 494, 175

Cimatti, A., Villani, D., Pozzetti, L., \& di Serego Alighieri, S. 2000, MNRAS, 318, 453

Clements, D. 2000, MNRAS, 312, 61

Dunlop, J., McClure, R., Kukula, M., et al. 2002, MNRAS, submitted [astro-ph/0108397]

Ellison, S. L. 2000, Ph.D. Thesis, University of Cambridge 
Ellison, S. L., Pettini, M., Steidel, C. C., \& Shapley, A. E. 2001a, ApJ, 549, 770

Ellison, S. L., Yan, L., Hook, I., et al. 2001b, A\&A, 379, 393

Elvis, M. 2000, ApJ, 545, 63

Finn, R., Impey, C., \& Hopper, E. 2001, ApJ, in press [astro-ph/0104124]

Foltz, C. B., Weymann, R. J., Peterson, B. M., et al. 1988, ApJ, 307, 504

Foltz, C. B., Chaffee, F. H., Weymann, R. J., \& Anderson, S. F. 1988, Proceedings of the QSO Absorption Line Meeting, 53

Fynbo, J. U., Møller, P., \& Warren, S. J. 1999, MNRAS, 305, 849

Gehrels, N. 1986, ApJ, 303, 336

Kellermann, K. I., Sramek, R., Schmid, M., Shaffer, D. B., \& Green, R. 1989, AJ, 98, 1195

Kurk, J. D., Venemans, B., Rottgering, H. J. A., Miley, G. K., \& Pentericci, L. 2001, Sesto conference proceeding Probing cosmic evolution with galaxy clusters [astro-ph/0110131]

Hall, P., \& Green, R. 1998, ApJ, 507, 558

Hall, P., Green, R., \& Cohen, M. 1998, ApJS, 119, 1

Hamilton, T., Casertano, S., \& Turnshek, D. A. 2001 [astro-ph/0011255]

Hamann, F., Barlow, T., \& Junkkarinen, V. 1997, ApJ, 478, 87

Hutchings, J. B. 2002, in QSO hosts and their environments, ed. I. Marquez (IAA, Granada, Spain) [astro-ph/0107157]

Hutchings, J. B., Crampton, D., \& Johnson, A. 1995, AJ, 109, 73

Hutchings, J. B., Crampton, D., Morris, S. L., Durand, D., \& Steinbring, E. 1999, AJ, 117, 1109

Jackson, C. A., Wall, J. V., Shaver, P. A., et al. 2002, A\&A, submitted

Lacy, M., Laurent-Muehleisen, S. A., Ridgway, S. E., Becker, R. H., \& White, R. L. 2001, ApJ, 551, L17

Lanzetta, K. M., McMahon, R. G., Wolfe, A. M., et al. 1991, ApJS, 77, 1

Lanzetta, K. M., Wolfe, A. M., \& Turnshek, D. A. 1995, 440, 435

Magorrian, J., Tremaine, S., Richstone, D., et al. 1998, AJ, 115,2285

Martínez, H. J., Merchan, M. E., Valotto, C. A., \& Lambas, D. G. 1999, ApJ, 514, 558

McLure, R. J., \& Dunlop, J. S. 2001, MNRAS, 321, 515
McLure, R. J., Kukula, M. J., Dunlop, J. S., et al. 1999, MNRAS, 308, 377

Møller, P., \& Warren, S. J. 1996, A\&A, 311, 25

Møller, P., Warren, S. J., \& Fynbo, J. U. 1998, A\&A, 330, 19

Monier, E., Mathur, S., Wilkes, B., \& Elvis, M. 2001, ApJ, submitted [astro-ph/0102348]

Morton, D., Wright, A. E., Peterson, B. A., Jauncey, D. L., \& Chen, J. 1980, MNRAS, 193, 339

Murdoch, H. S., Hunstead, R. W., Pettini, M., \& Blades, J. C. 1986, ApJ, 309, 19

Pascarelle, S., Lanzetta, K., Chen, H.-W., Webb, J. 2001, ApJ, in press [astro-ph/0107295]

Pentericci, L., Kurk, J. D., Rottgering, H. J. A., et al. 2000, A\&A, 361, L25

Péroux, C., Storrie-Lombardi, L. J., McMahon, R. G., Irwin, M., \& Hook, I. M. 2001, AJ, 121, 1799

Petitjean, P., Rauch, M. J., \& Carswell, R. F. 1994, A\&A, 291, 29

Richards, G. T. 2001, ApJS, 133, 53

Richards, G. T., Laurent-Muehleisen, S. A., Becker, R., \& York, D. G. 2001, 547, 635

Richards, G. T., York, D. G., Yanny, B., et al. 1999, ApJ, 513, 576

Sanchez, S., \& Gonzalez-Serrano, J. 1999, A\&A, 352, 383

Stocke, J. T., Morris, S. L., Weymann, R. J., Foltz, C. B. 1992, ApJ, 396, 487

Storrie-Lombardi, L., \& Wolfe, A. M. 2000, ApJ, 543, 552

Teplitz, H., MacLean, I., \& Malkan, M. 1999, ApJ, 520, 469

Warren, S., Møller, P., Fall, S. M., \& Jakobsen, P. 2001, MNRAS, in press [astro-ph/0105032]

White, R., Becker, R. H., Gregg, M. D., et al. 2000, ApJS, 126, 133

Wold, M., Lacy, M., Lilje, P., \& Serjeant, S. 2001, MNRAS, 323,231

Wolfe, A. M., Lanzetta, K. M., Foltz, C. B., \& Chaffee, F. H. 1995, ApJ, 454, 698

Wolfe, A. M., Turnshek, D. A., Smith, H. E., \& Cohen, R. D. 1986, ApJS, 61, 249

Yamada, T., Tanaka, I., Aragon-Salamanca, A., et al. 1997, ApJ, 487, 125

Yee, H., \& Green, R. 1987, AJ, 94, 618 